\title{
Müller Cells Stabilize Microvasculature through Hypoxic Preconditioning
}

\author{
Kepeng Ou $u^{a, b}$ Sonja Mertsch ${ }^{a}$ Sofia Theodoropoulou ${ }^{b}$ Jiahui $\mathrm{Wu}^{b}$ \\ Jian Liu ${ }^{\mathrm{b}}$ David A. Copland ${ }^{\mathrm{b}}$ Louis M. Scott ${ }^{\mathrm{b}}$ Andrew D. Dick ${ }^{\mathrm{b}, \mathrm{c}}$ \\ Stefan Schrader ${ }^{\mathrm{a}, \mathrm{d}}$ Lei Liu ${ }^{\mathrm{b}}$
}

aLaboratory for Experimental Ophthalmology, University of Düsseldorf, Düsseldorf, Germany, ${ }^{\mathrm{b} A c a d e m i c}$ Unit of Ophthalmology, Bristol Medical School, University of Bristol, Bristol, UK, 'National Institute for Health Research (NIHR) Biomedical Research Centre at Moorfields Eye Hospital and University College London Institute of Ophthalmology, London, UK, 'Department of Ophthalmology, University of Düsseldorf, Düsseldorf, Germany

\section{Key Words}

Müller cells • Angiogenesis • Vascular permeability • Hypoxia • Hypoxic preconditioning

\begin{abstract}
Background/Aims: Hypoxia of the retina is a common pathogenic drive leading to vision loss as a result of tissue ischemia, increased vascular permeability and ultimately retinal neovascularisation. Here we tested the hypothesis that Müller cells stabilize the neurovascular unit, microvasculature by suppression of HIF- $1 \alpha$ activation as a result of hypoxic preconditioning. Methods: Tube Formation Assay and In vitro Vascular Permeability Image Assay were used to analyze angiogenesis and vascular integrity. Seahorse XF Cell Mito Stress Test was used to measure mitochondrial respiration. Gene and protein expression were examined by qRTPCR, ELISA and western blot. Results: Hypoxic insult induces a significant induction of proangiogenic factors including vascular endothelial growth factor (VEGF) and angiopoietinlike 4 (ANGPTL-4) resulting in angiogenesis and increased vascular permeability of vascular endothelial cells. Hypoxic preconditioning of a human retinal Müller glia cell line significantly attenuates HIF- $1 \alpha$ activation through the inhibition of mTOR and concomitant induction of aerobic glycolysis, stabilizing endothelial cells. Conclusion: Hypoxic preconditioning of Müller cells confers a robust protection to endothelial cells, through the suppression of HIF$1 \alpha$ activation and its downstream regulation of VEGF and ANGPTL-4.
\end{abstract}




\section{Cellular Physiology Cell Physiol Biochem 2019;52:668-680 \\ \begin{tabular}{ll|l} 
and Biochemistry & $\begin{array}{l}\text { DOI: 10.33594/000000047 } \\
\text { Published online: } 29 \text { March } 2019\end{array}$ & $\begin{array}{l}\text { C } 2019 \text { The Author(s). Published by } \\
\text { Cell Physiol Biochem Press GmbH\&Co. KG }\end{array}$
\end{tabular} \\ Ou et al.: Microvasculature Stabilization through Hypoxic Preconditioning}

\section{Introduction}

Retinal hypoxia is recognized in a number of sight-threatening disorders including central retinal artery occlusion, ischemic central retinal vein occlusion, diabetic retinopathy and occlusive retinal vasculitis [1-3]. Hypoxic environments induce expression of Hypoxiainducible factor 1 -alpha (HIF-1 $\alpha$ ), which modulates target genes such as VEGF and nitric oxide synthase (NOS) in many tissues [4]. Under conditions of hypoxic stress, degradation of the oxygen-sensitive HIF-1 $\alpha$ subunit is reduced, whereas its transcriptional activity is enhanced [5-7]. The increased amount of active HIF-1 $\alpha$ protein localizes to the nucleus by binding to HIF-1 $\beta$, forming a heterodimer (HIF-1) that is capable of binding to the DNA of specific (hypoxia-inducible) genes and inducing broad changes in gene expression, which mediate acclimation of cells, tissues, and the organism to conditions of low oxygen tension [8]. The HIF family consists of three identified members, with HIF-1 being the best-characterized protein [9]. The action of HIF-1 and associated downstream regulated genes, particularly VEGF, are essential for retinal development, vasculature stability, proper retinal function, and vision maintenance [10]. Nevertheless, the HIF-1 targeted genes represent a doubleedged sword [11]. Under hypoxic conditions, overexpression of these target genes alters the tissue homeostasis and contribute to ocular disease, through increased retinal angiogenesis and vascular permeability [12].

Müller cells account for $90 \%$ of the retinal glia [13]. Müller cells span across the entire thickness of the retina, from the inner limiting membrane of the retina to the distal end of the outer nuclear layer. Anatomically and functionally Müller cells constitute a link between the retinal neurons, the vitreous body, the retinal blood vessels (forming glial limitans) and the subretinal space [14]. However, reactive Müller cell gliosis often occurs in the mammalian retina in response to injury. It has been demonstrated that the release of several proinflammatory factors, cytokines and proangiogenic growth factors from Müller cells can accentuate retinal damage [15]. As an example, evidence demonstrates that VEGF and ANGPTL-4 are up-regulated by HIF-1 in hypoxic retinal Müller cells in vitro and the ischemic inner retina in vivo [16]. Increased expression of VEGF and ANGPTL-4 promotes angiogenesis and vascular permeability resulting in disruption of the blood retinal barrier [16][17].

Hypoxic preconditioning (HP) or hypoxia-induced tolerance, refers to a brief period of hypoxia that induces protection against subsequent lethal insult occurring minutes, hours, or even days later [18]. HP represents a fundamental adaptive response to environmental stress, whereby cells adapt to stress by upregulating defense mechanisms and switching to a protective phenotype [19]. Reports have shown that HP stabilizes HIF-1 $\alpha$ in the retina and protects photoreceptors against light-induced cell death [20]. Whilst the underlying pro-survival mechanisms are not fully understood, the cellular response to hypoxia is characterized by the differential regulation of genes. Therefore, we hypothesized that the beneficial effects of HP might involve counter-regulation of Müller cell activation and negate subsequent secretion of pro-angiogenic factors. In this in vitro study, we demonstrate that HP of Müller cells stabilizes microvasculature via HIF-1 regulation.

\section{Materials and Methods}

\section{Cell Culture and Reagents}

Spontaneously immortalized human Müller glia cell line (MIO-M1) purchased from UCL Business PLC (London, UK), were cultured in DMEM medium containing high glucose and stable glutamine, supplemented with 10\% fetal bovine serum (FBS) (Life Technologies, UK). Human Primary Retinal Microvascular Endothelial cells (HRMECs, H6065, Generon, UK) were cultured in Complete Human Endothelial Cell Medium (H1168, Generon, UK), Human Umbilical Vein Endothelial Cells (HUVECs) (C-12200, Promocell, UK) were cultured in Endothelial Cell Growth Medium 2 (C-39211, Promocell, UK). Rapamycin (R8781, working concentration $100 \mathrm{nM}$ ) and Digoxin (D6003, working concentration $100 \mathrm{nM}$ ) were obtained from 


\section{Cellular Physiology Cell Physiol Biochem 2019;52:668-680 \\ \begin{tabular}{ll|l} 
DOl: 10.33594/000000047 & O 2019 The Author(s). Published by \\
Cell Physiol Biochem Press GmbH\&Co. KG
\end{tabular} \\ Ou et al.: Microvasculature Stabilization through Hypoxic Preconditioning}

Sigma-Aldrich. Apoptosis/ Necrosis Detection Kit (ab176749, Abcam, UK) was used to assess HP-induced cell viability. MIO-M1 were seeded at a density of 3x $10^{4}$ in 96 well plate, then cultured in normoxic and hypoxic incubator for 3 days. Cells were then stained with Apoptosis/ Necrosis Detection Kit as described by the manufacturer's protocol. MIO-M1 cell viability was quantified by counting the number of apoptosis and necrosis cells account for the total number of cells under a fluorescence microscope (Nikon, Japan).

Hypoxic conditions was used according to previous report [16]. In brief, MIO-M1 cells were cultured in normal conditions to confluency and then transferred to hypoxic chamber supplied with $1 \% \mathrm{O}_{2}, 94 \% \mathrm{~N}_{2}$, and $5 \% \mathrm{CO}_{2}$. Control cultures were incubated under standard normoxic conditions for the same duration. In HP experiments, MIO-M1 cells are transferred to hypoxic culture conditions for a defined duration (1 hour, 2 hours and 4 hours) before returning and reoxygenation in the normoxic incubator (95\% air, 5\% $\mathrm{CO}_{2}$ ) for 24 hours. Cultures are then re-incubated for a further 48 hours within the hypoxic environment. After the re-incubation time, the supernatant and cells were collected for the quantitative assays.

\section{Quantitative Real time RT-PCR}

Total RNA extraction was carried out using TRIzol ${ }^{\mathrm{TM}}$ Reagent (15596026, Life Technologies, UK) according to the manufacturer's instructions. Total RNA was quantified by Nanodrop and treated with RQ1 RNase-free DNase before cDNA synthesis using the ImProm-II ${ }^{\mathrm{TM}}$ Reverse Transcription System (A3800, Promega, UK). cDNA was amplified using the Power SYBR® Green PCR Master Mix Reagent (4367659, Life Technologies, UK) on a StepOne ${ }^{\mathrm{TM}}$ Applied Biosystems Real-Time PCR System. Primer sequences used were: $\beta$-Actin, forward 5'-gggaaatcgtgcgtgacattaag, reverse 5'-tgtgttggcgtacaggtctttg; HIF-1 $\alpha$, forward 5'-gaaagcgcaagtcctcaaag, reverse 5'-tgggtaggagatggagatgc; ANGPTL-4, forward 5'-ggacacggcctatagcctg, reverse 5'-ctcttggcgcagttcttgtc. succinate dehydrogenase complex subunit D (SDHD), forward 5'-atggcggttctctggaggctg, reverse 5'-gagcttccacgcatggcaac; glucose transporter 1 (GLUT1), forward 5 '-tcactgtgctcctggttttctg, reverse $5^{\prime}$-cctgtgcctcctgagagatcc. The equation fold change $=2^{-\Delta \Delta c t}$ was used for calculation relative changes in expression levels. All measurements were performed at least in duplicates and the experiments were performed at least three times independently.

\section{SDS_PAGE analysis}

For analysis of protein levels, cultured cells were rinsed twice with ice-cold PBS and then lysed with ice-cold CelLytic ${ }^{\mathrm{TM}}$ MT Cell Lysis Reagent (C2978, Sigma-Aldrich, UK). Cell lysates were centrifuged at 14, $000 \mathrm{~g}$ for $10 \mathrm{~min}$ at $4^{\circ} \mathrm{C}$, the supernatant was transferred to fresh tubes and Pierce ${ }^{\mathrm{TM}}$ BCA Protein Assay Kit (23225, Thermo Fisher Scientific, UK) used to determine the protein concentration in each sample. Then the equal protein was mixed with $4 \mathrm{x}$ SDS sample buffer, boiled for $10 \mathrm{~min}$ at $90^{\circ} \mathrm{C}$, and resolved using $4 \%$ to 20\% NuPAGE gels (XV04200PK20, Invitrogen, UK). After electrophoresis, the proteins were transferred to PVDF membranes (IB24001, Invitrogen, UK) by electrophoretic transfer. The membranes were blocked with $5 \%$ skimmed milk for 2 hours, rinsed, and incubated overnight at $4{ }^{\circ} \mathrm{C}$ with the following primary antibodies: Anti-HIF-1 alpha (ab2185; Abcam, UK; 1: 1000) and mTOR (7C10) Rabbit mAb (2983; Cell Signaling Technology, UK; 1:1000). Excess antibody was then removed by washing the membrane in PBS/ $0.1 \%$ Tween 20, and the membranes were incubated for 2 hours with horseradish peroxidase-conjugated secondary antibodies (A0545, Abcam, UK; 1:2000). Following further washes in PBS/ 0.1\% Tween 20, the signals were developed with ECL reagent (GERPN2209, Sigma, UK) and captured by an electronic imaging system (Konica Minolta).

\section{ELISA}

To quantify levels of secreted VEGF and ANGPTL-4, culture supernatants from hypoxic conditioned MIO-M1 cells were assayed using DuoSet human VEGF (DY293B, Bio-Techne, UK) or ANGPTL-4 ELISA (DY3458, Bio-Techne, UK) kits according manufacturer's instructions. Three samples for each experimental condition were used. In each experiment, all samples and standards were measured in duplicate.

\section{Tube formation}

Tube formation assay was performed using growth factor-reduced Matrigel (No.354320, BD Biosciences, UK). Fifty microliters of Matrigel was added into a prechilled 96 -well plate and placed in a $37^{\circ} \mathrm{C}$ $\mathrm{CO}_{2}$ incubator for $30 \mathrm{~min}$. HRMECs were counted and seeded $2 \times 10^{4}$ cells in $100 \mu \mathrm{l}$ of culture medium which were mixed with $50 \mu \mathrm{l}$ of test sample, then added onto the matrigel surface. Eighteen hours later, phase 


\section{Cellular Physiology Cell Physiol Biochem 2019;52:668-680 \\ \begin{tabular}{ll|l} 
and Biochemistry & $\begin{array}{l}\text { DOl: 10.33594/000000047 } \\
\text { Published online: 29 March 2019 }\end{array}$ & $\begin{array}{l}\text { O 2019 The Author(s). Published by } \\
\text { Cell Physiol Biochem Press GmbH\&Co. KG }\end{array}$ \\
\cline { 2 - 3 } &
\end{tabular} \\ Ou et al.: Microvasculature Stabilization through Hypoxic Preconditioning}

contrast photos were taken by Wide field microscope (Leica DMI6000). The whole area of tube length was quantified by ImageJ1.46r (National Institutes of Health, USA).

\section{Permeability Assay}

Permeability visualization experiments were performed on $18 \times 18 \mathrm{~mm}$ square Rinzle plastic coverslips with In vitro Vascular Permeability Imaging Assay (17-10398, Merck, UK). HUVEC cells were cultured for 48-72 hours on biotinylated gelatin coated coverslips in $35 \mathrm{~mm}$ culture dishes in a density of $4.5 \times 10^{5}$ cells/ dish. The culture medium was then changed to EGM-2 containing 2\% FBS for 2 hours prior to stimulation. Then culture medium was mixed with test samples at $1: 3$ ratio and incubated for $30 \mathrm{~min}$ at $37^{\circ} \mathrm{C}$ before evaluation of HUVEC cell monolayer permeability. FITC-streptavidin $(25 \mu \mathrm{g} / \mathrm{ml})$ was directly added to the culture medium for $5 \mathrm{~min}$, followed by two washing steps ( $3 \mathrm{ml}$ of $\mathrm{PBS}, \mathrm{pH} 7.4,37^{\circ} \mathrm{C}$ ). The cells were fixed ( $3.7 \%$ formaldehyde in PBS for $10 \mathrm{~min}$ ) and subjected to immunofluorescence staining for VE-cadherin according to the manufacturer's instructions. Overlay of FITC and VE-cadherin staining demonstrates reciprocal relations between VE-cadherin peripheral localization and increased local permeability of FITCstreptavidin. Local permeability change induced by conditioned medium was visualized in cells grown on biotinylated gelatin plastic coverslips. The pattern of FITC-streptavidin binding to the biotinylated gelatin underlying the cell monolayer was examined under Leica SP5-AOBS confocal laser microscope. Images were processed with ImageJ software.

\section{Extracellular flux analysis}

Cell metabolism was assessed using a Seahorse XFp Extracellular Flux Analyzer (Agilent, UK). MIO-M1 cells were seeded at a density of $3 \times 10^{4}$ per well and were treated with appropriate reagents. Real time measurements of oxygen consumption rate (OCR) were normalized to total protein content using a BCA assay. Pre-optimized injections of the reagents specific for each assay were used. Cell Mito Stress kit (103015-100, Agilent, UK) injections: oligomycin, FCCP and antimycin A/rotenone.

\section{Statistical Analysis}

Results from cell culture and animal models are shown as mean \pm SD from a minimum of three independent experiments. Statistical analysis was performed with one-way ANOVA with Dunn's test for multiple comparisons, while unpaired Student's $t$ test and Mann-Whitney test for two individual comparisons. where indicated. Statistical analysis was performed using Prism 6.0 software (Graph- Pad). ${ }^{*} P$ $<0.05 ;{ }^{* *} P<0.01 ;{ }^{* * *} P<0.001 ;{ }^{* * * *} P<0.0001$.

\section{Results}

To determine whether HP can alter MIO-M1 cells responses in low-level oxygen environments, we initially established the hypoxic response profile of the MIO-M1 cell line through in vitro assays. Consistent with previous reports [16-21], MIO-M1 cells remain viable when cultured in a hypoxic $\left(1 \% \mathrm{O}_{2}\right)$ environment, with increased protein expression of HIF$1 \alpha$, and gene expression and secretion of VEGF and ANGPTL- 4 over the 48 hour time-period examined (Fig. 1). Pretreatment of MIO-M1 cells with digoxin to block hypoxic induction of HIF-1 $\alpha$, suppressed VEGF and ANGPTL-4 production (Fig. 1).

To confirm a potential contribution of secreted factors elicited by hypoxia-treated MIO-M1 cells to angiogenesis and vascular leakage, HRMECs were treated with conditioned medium from the MIO-M1 cells exposed to hypoxia for 48 hours (Hypoxia-CM). Endothelial cell proliferation and permeability was determined by tube formation and FITC-positive intercellular signal respectively. Hypoxia-CM increased HRMEC tube length compared with normoxic conditioned medium (Normoxia-CM). Conditioned medium from hypoxic MIO-M1 cells pre-treated with digoxin resulted in reduced overall tube length, comparable to Normoxia-CM (Fig. 2A-B). Furthermore, exposure of a monolayer of HRMECs to HypoxiaCM from the MIO-M1 cells promoted endothelial cell permeability and was perturbed by addition of digoxin (Fig. 2C-D). Collectively, these results support a HIF- $1 \alpha$-mediated upregulation and functional effect of pro-angiogenic factors secreted by MIO-M1 cells. 


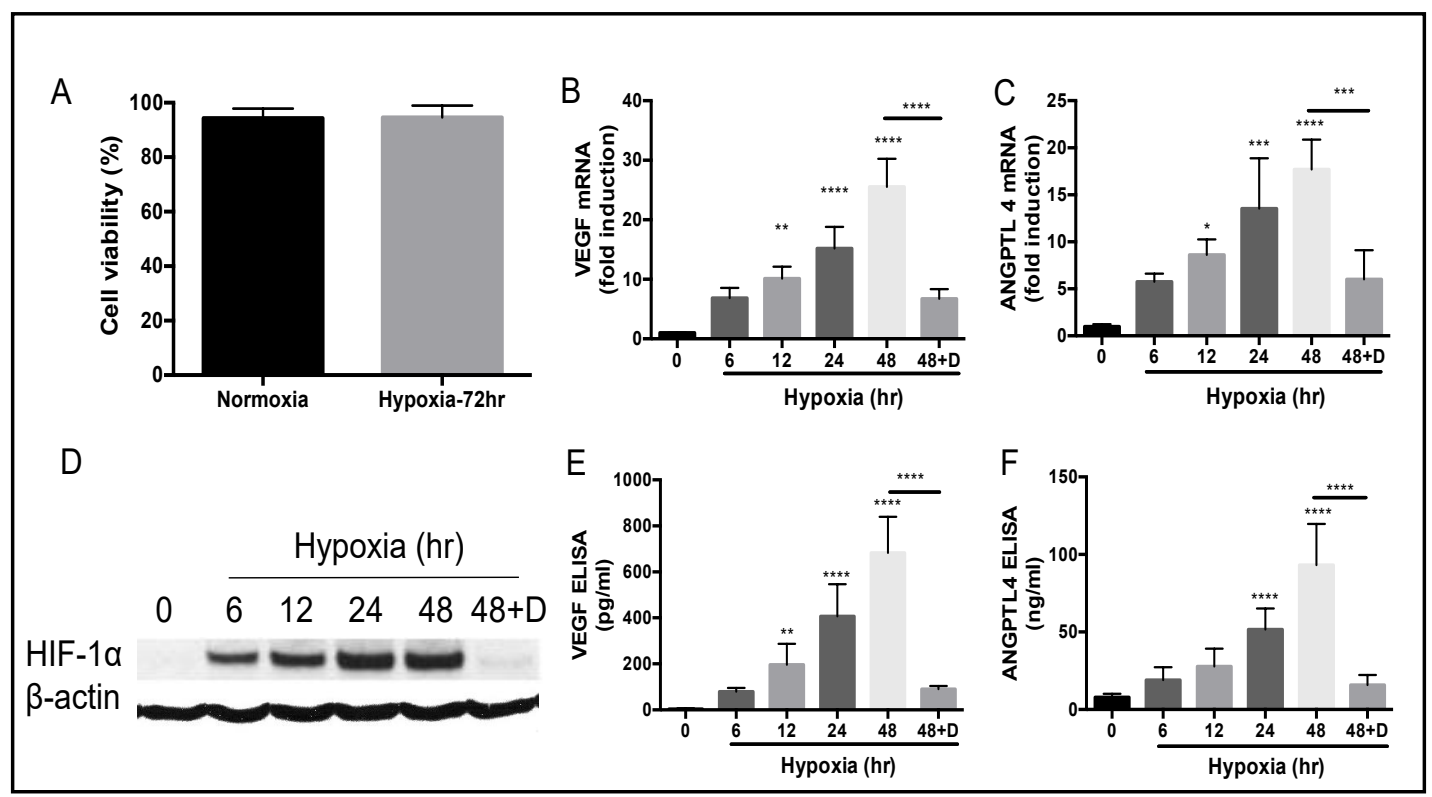

Fig. 1. Hypoxia insult induces HIF-1-dependent up-regulation of VEGF and ANGPTL-4 in cultured MIO-M1 cells without reducing cell viability. (A). Prolonged $1 \% \mathrm{O}_{2}$ hypoxia (72 hours) did not alter the cell viability staining in MIO-M1 cells culture ( $\mathrm{n}=4$ per group). (B-F) Exposure of MIO-M1 cells to hypoxia (indicated time) resulted in an increase of HIF-1 $\alpha$ protein and a corresponding increase in VEGF and ANGPTL-4 mRNA and protein level, which can be inhibited by pretreatment with the HIF inhibitor, digoxin (100 nM; 2 hours before hypoxia, 48+D) ( $n=4$ per group). Data represents means \pm SD of relative values vs control from 3 independent experiments. ${ }^{*} \mathrm{P}<0.05 ;{ }^{* *} \mathrm{P}<0.01$; ${ }^{* *} \mathrm{P}<0.001$; ${ }^{* * *} \mathrm{P}<0.0001$, statistical analysis was performed with one-way ANOVA with Dunn's test for multiple comparisons, while unpaired Student's t test and MannWhitney test for two individual comparisons.

Extending these observations further to determine a suitable hypoxic stimulus period for evaluating HP responses, we wished to confirm the early hypoxic response kinetics of HIF expression. MIO-M1 cells were similarly cultured in low $\mathrm{O}_{2}$ conditions, mRNA and protein expression levels were quantified at several early time points, ranging from 1 to 6 hours. Expression of HIF, VEGF and ANGPTL-4 increased in a time-dependent response; elevated by 2.6 folds by 2 hours, and further increased by 3.9 folds by 4 hours, as compared to cells under normoxic conditions (Fig. 3A-E). Based on the concept of HP (brief hypoxic exposure), we determined that the cellular response within the first 6 hours following hypoxic exposure elicit induced HIF \& proangiogenic expression, but not to a detrimental level. On this basis, we examined the effect of $1,2 \& 4$ hours HP exposure to assess the potential beneficial effect of HP.

Hypoxic Preconditioning Suppresses Hypoxia Induced Expression of HIF-1 $\alpha$, AngiopoietinLike 4 and VEGF in MIO-M1 cells

The HP protocol involves an initial short exposure of MIO-M1 cells to hypoxia (1, 2 or 4 hours), then a 24 hours reoxygenation period under standard normoxic conditions, before a return to the hypoxic environment for a further 48 hours (Supplementary Fig. 1 - all supplementary material available online at www.cellphysiolbiochem.com). We compared the effect of the HP duration on VEGF and ANGPTL-4 expression to MIO-M1 cells without return to normoxia. Following HP for 2 hours, the level of mRNA and secreted protein were both significantly reduced. HP for 1 hour and 4 hours did not alter expression (Fig. 4A-D). To interrogate whether the protective effect of HP in MIO-M1 cells in hypoxia was mediated via altered expression/regulation of HIF-1 $\alpha$, we performed SDS-PAGE on cell lysates from HP and hypoxia alone samples. Consistent with our angiogenic markers expression, HP-2 hours resulted in the maximal attenuation of HIF-1 $\alpha$ protein accumulation (Fig. 4E). 


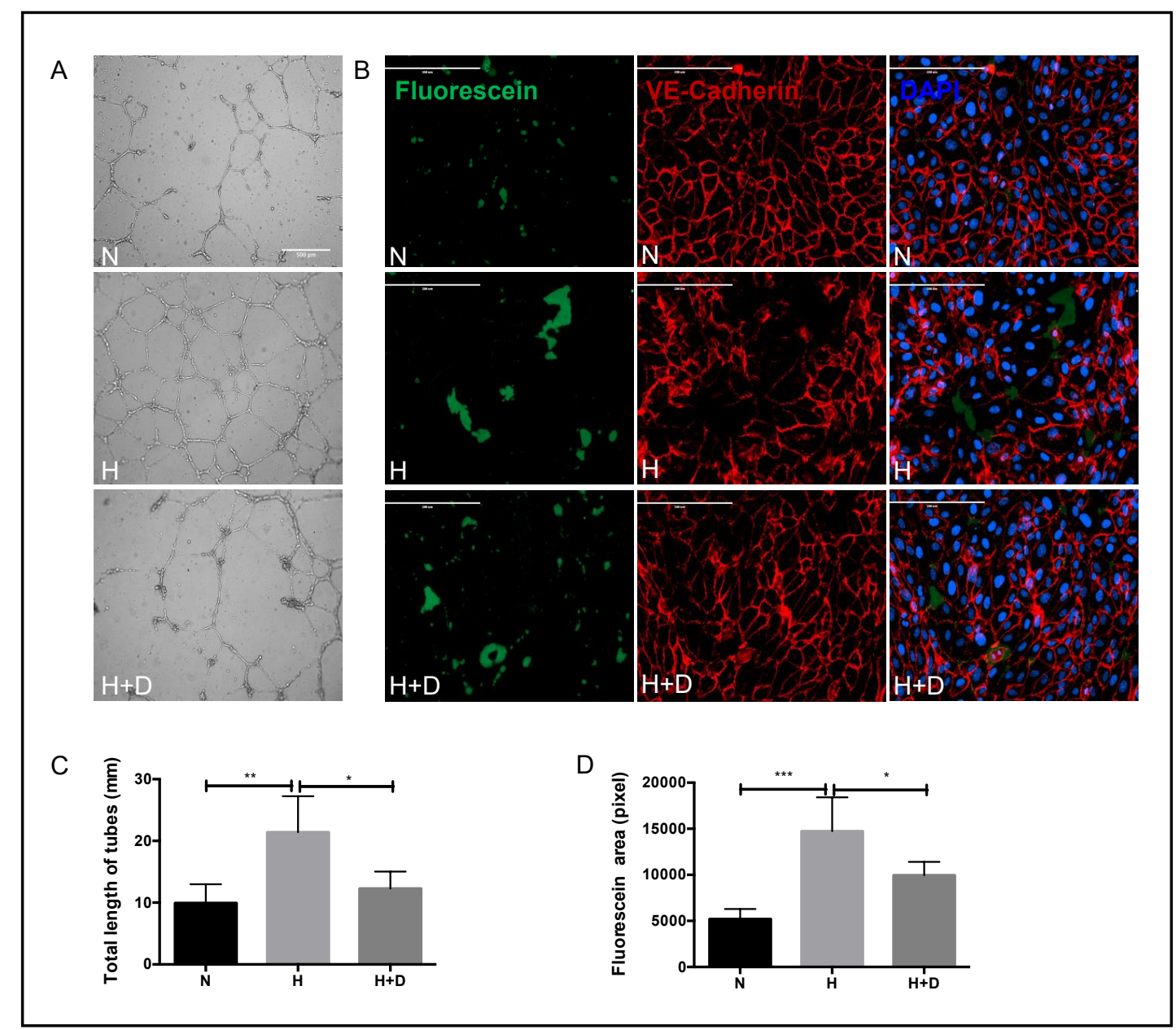

Fig. 2. Conditioned medium from hypoxic MIO-M1 cells promotes angiogenesis and vessel leakage. (AB) Conditioned media from MIO-M1 cells exposed to $1 \% \mathrm{O}_{2}$ hypoxia 48 hours $(\mathrm{H})$ stimulated HRMECs tube formation compared to $20 \% \mathrm{O}_{2}$ normoxic condition $(\mathrm{N})(\mathrm{n}=12$ per group). The total length of tubes decreased by pretreatment with digoxin $(\mathrm{H}+\mathrm{D})$. Images was performed with Leica DMI6000 microscope (x5). Scale bar, $500 \mu \mathrm{m}$. (C-D) The area of FITC-positive intercellular spots increased conditioned medium from hypoxic MIO-M1 cells and decreased by pretreatment with digoxin ( $\mathrm{n}=3$ per group). Scale bar, 200 $\mu \mathrm{m}$. Data represents means $\pm \mathrm{SD}$ of relative values vs control from 3 independent experiments. ${ }^{*} \mathrm{P}<0.05$; ${ }^{* *} \mathrm{P}<0.01 ;{ }^{* * *} \mathrm{P}<0.001 ;{ }^{* * * *} \mathrm{P}<0.0001$, statistical analysis was performed with one-way ANOVA with Dunn's test for multiple comparisons.

\section{Hypoxic Preconditioning Suppresses Hypoxia Induced Angiogenesis and Vascular} Permeability in MIO-M1 cells

The data demonstrates the potential beneficial effect of HP that suppresses HIF mediated upregulation of proangiogenic factors in response to hypoxia. Thus, we evaluated how altered cellular responses following HP may regulate endothelial cell responses. Monolayers of HRMECs were treated with Hypoxia-CM or conditioned medium from hypoxic preconditioning (Hypoxic preconditioning-CM) and tube formation and vascular permeability assessed. Whilst Hypoxia-CM promoted endothelial cell proliferation, Hypoxic preconditioning-CM from MIO-M1 cells subjected to the HP-2 hours protocol demonstrate significantly reduced HRMEC tube length and junction formation by $35 \%$ and $64 \%$ respectively (Fig. 5A-C). The HP-1 hour and HP-4 hours conditions failed to significantly reduce angiogenic potential. Similarly, assessment of vascular permeability corroborates the anti-angiogenic potential that HP confers to MIO-M1 cells. Conditioned medium from the MIO-M1 cells exposed at HP-2 hours demonstrate reduced by $44 \%$ endothelial cell permeability with decreased FITC- 


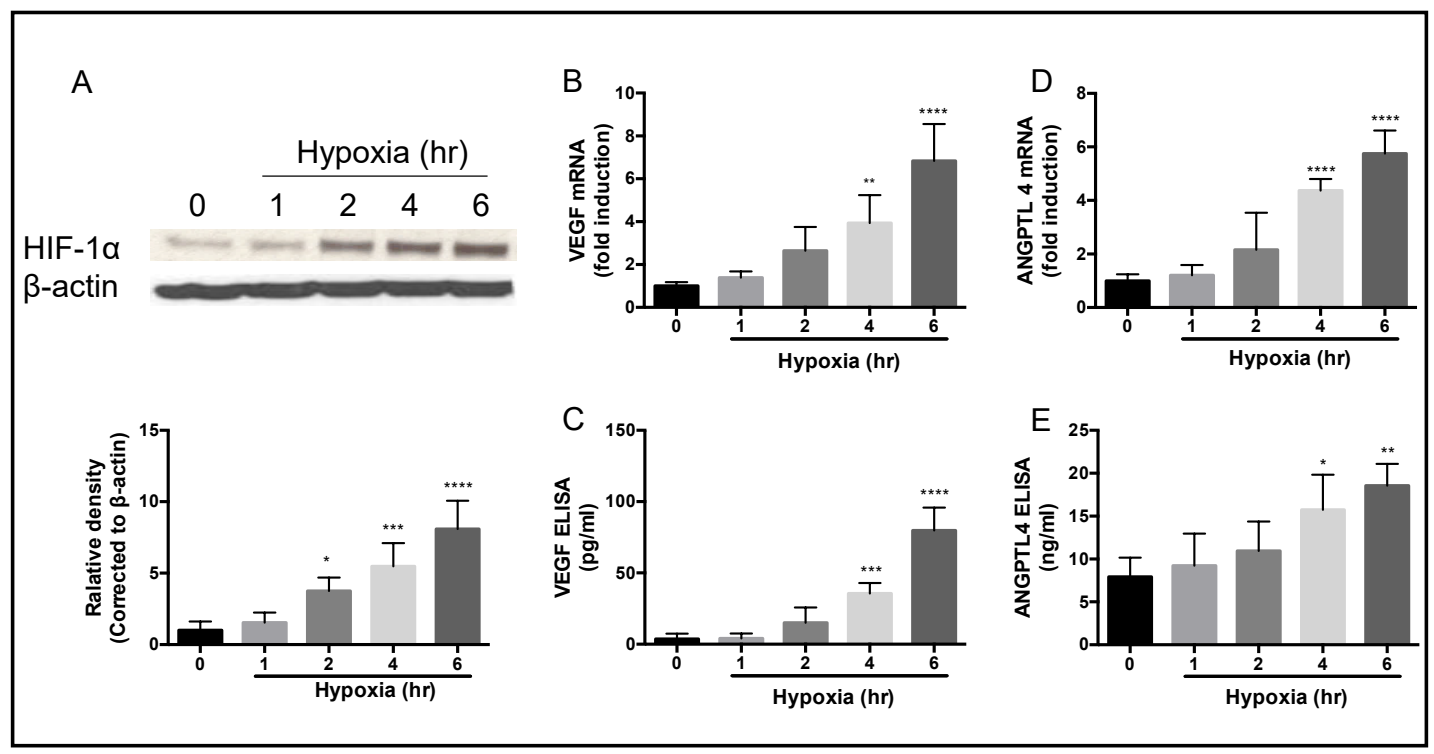

Fig. 3. HIF- $1 \alpha$ protein accumulation and VEGF expression in cultured hypoxic MIO-M1 cells. (A) Exposure of MIO-M1 cells to $1 \% \mathrm{O}_{2}$ hypoxia (indicated time) induces HIF- $1 \alpha$ protein stability. (B-E) Hypoxic conditions lead to increase expression of VEGF and ANGPTL-4 mRNA and protein. All the samples were normalized to their $\beta$-actin expression ( $n=4$ per group). Data represents means \pm SD of relative values vs control from 3 independent experiments. ${ }^{*} \mathrm{P}<0.05 ;{ }^{* *} \mathrm{P}<0.01$; ${ }^{* * *} \mathrm{P}<0.001$; ${ }^{* * *} \mathrm{P}<0.0001$, statistical analysis was performed with one-way ANOVA with Dunn's test for multiple comparisons.

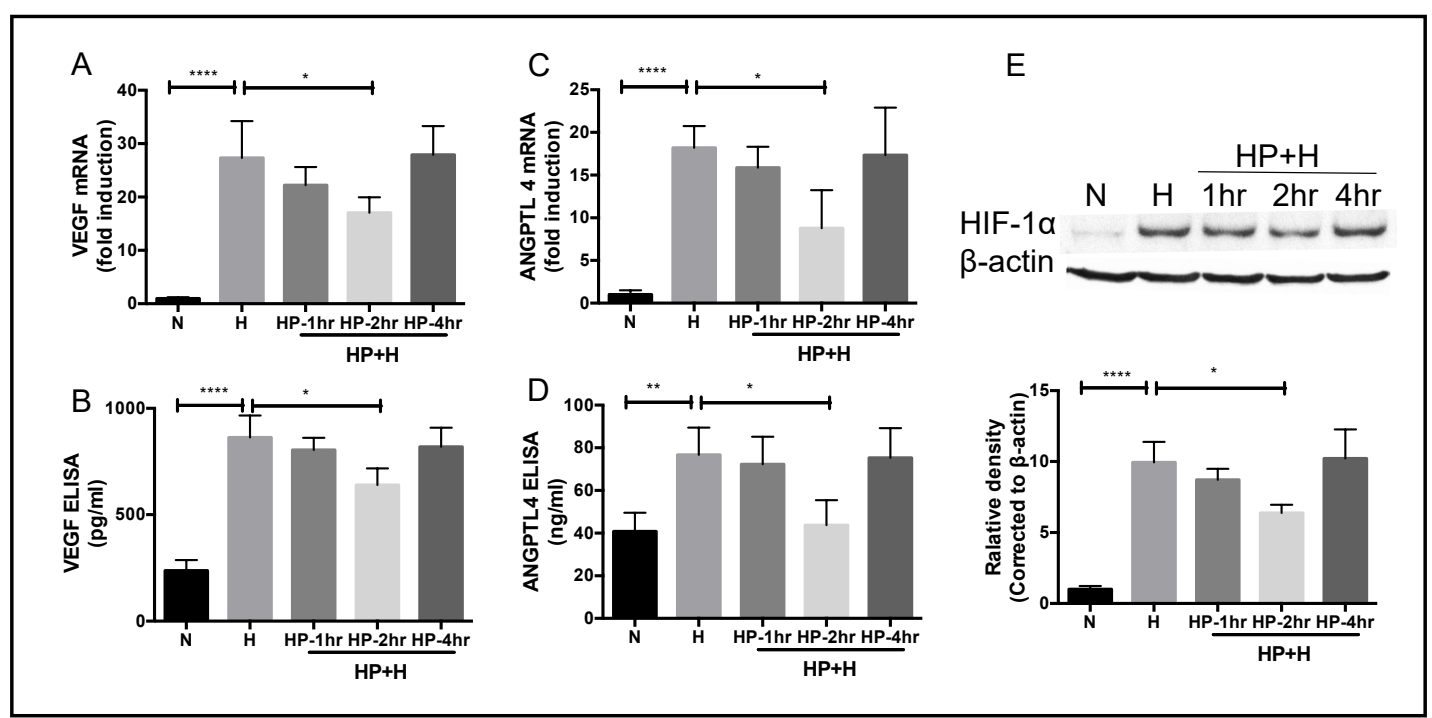

Fig. 4. Hypoxic preconditioning suppresses prolonged hypoxia-induced VEGF and ANGPTL-4 in MIO-M1 cells culture. (A-E) The expression of VEGF and ANTPGL-4 as well as HIF- $1 \alpha$ protein remarkably increased with exposure of MIO-M1 cells to $1 \% \mathrm{O}_{2}$ Hypoxia 48 hours $(\mathrm{H})$ in comparison with $20 \% \mathrm{O}_{2}$ normoxic conditions $(\mathrm{N})$. Brief hypoxia exposure for 2 hours before prolonged hypoxia $(\mathrm{HP}+\mathrm{H})$ insult significantly reduced the expression of VEGF and ANGPTL-4. The induction of HIF- $1 \alpha$ was also significantly suppressed by brief HP for 2 hours ( $n=4$ per group). Data represents means \pm SD of relative values vs control $(\mathrm{N})$ from 3 independent experiments. ${ }^{*} \mathrm{P}<0.05 ;{ }^{* * *} \mathrm{P}<0.001 ;{ }^{* * * *} \mathrm{P}<0.0001$, statistical analysis was performed with oneway ANOVA with Dunn's test for multiple comparisons. 


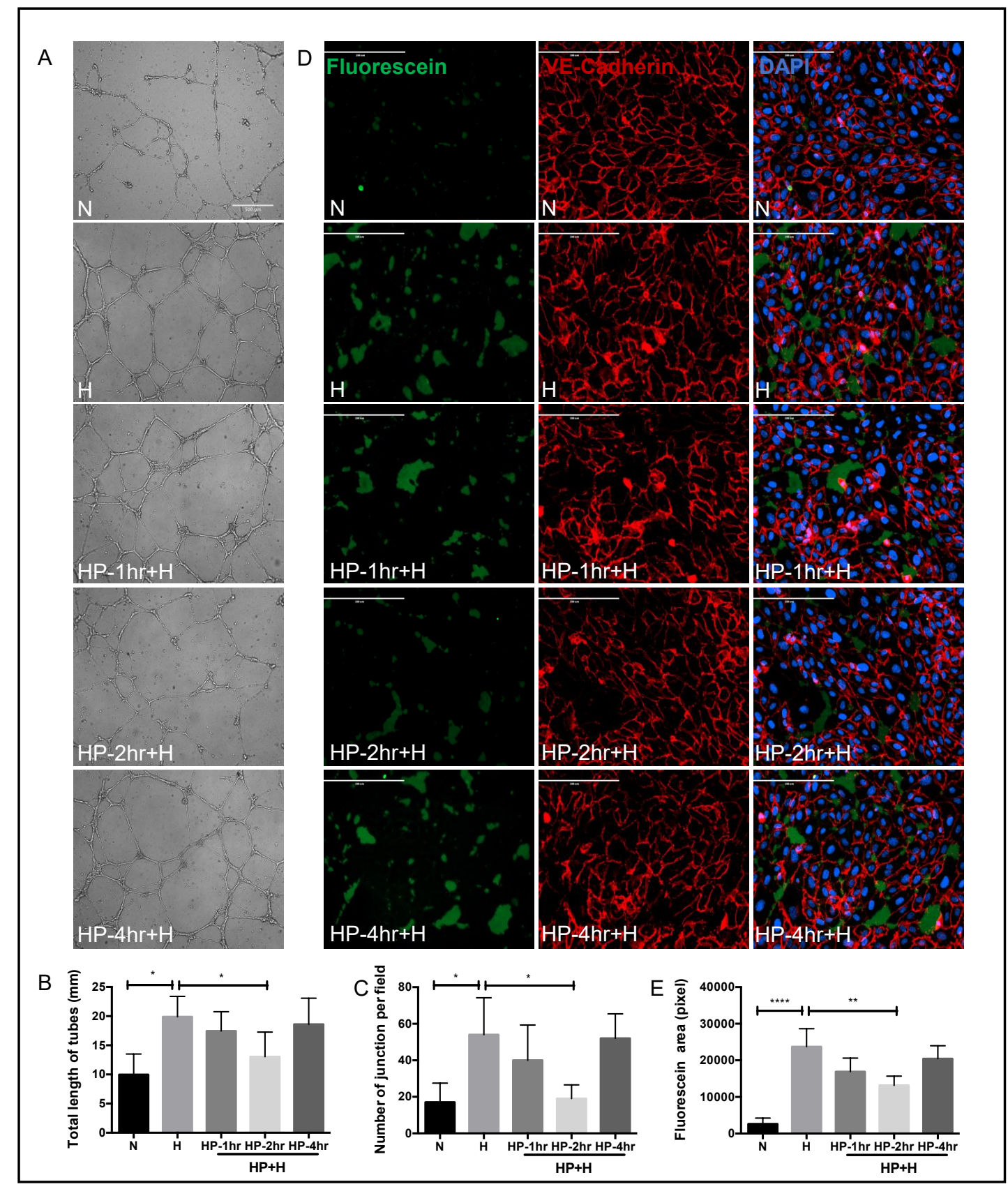

Fig. 5. Hypoxic preconditioning decreases HIF-1-dependent up-regulation of angiogenesis and vascular permeability. (A-C) Stimulation of HRMECs tube formation by conditioned media from MIO-M1 cells exposed to $1 \% \mathrm{O}_{2}$ hypoxia 48 hours $(\mathrm{H})$ compare to $20 \% \mathrm{O}_{2}$ normoxic condition $(\mathrm{N})$. Supernatant from HP- 2 hours (HP-2hr) reduced both the total length and junction number of tubes compared to Hypoxic group ( $\mathrm{n}=12$ per group). Images was performed with Leica DMI6000 microscope (x5). Scale bar, $500 \mu \mathrm{m}$. (D, E) HUVECs were plated on coverslips coated with biotinylated gelatin and grown to confluence. then treated for 10 min with conditioned medium from cultured muller cells. FITC-avidin was added for 3 min, cells were fixed and subjected to immunofluorescence staining for VE-cadherin (red) to visualize cell-cell contacts. Green fluorescence depicts areas permeable for FITC-labeled avidin. Conditioned medium from hypoxic MIO-M1 cells increase area of FITC-positive intercellular spots and decreased by HP-2 hours ( $\mathrm{n}=3$ per group). Scale bar, $200 \mu \mathrm{m}$. Data represents means \pm SD of relative values vs control from 3 independent experiments. ${ }^{*} \mathrm{P}<0.05$; ${ }^{* *} \mathrm{P}<0.01 ;{ }^{* * *} \mathrm{P}<0.001 ;{ }^{* * *} \mathrm{P}<0.0001$, statistical analysis was performed with one-way ANOVA with Dunn's test for multiple comparisons. 


\section{Cellular Physiology Cell Physiol Biochem 2019;52:668-680 \\ \begin{tabular}{ll|l} 
and Biochemistry & $\begin{array}{l}\text { DOl: 10.33594/000000047 } \\
\text { Published online: 29 March 2019 }\end{array}$ & $\begin{array}{l}\text { O } 2019 \text { The Author(s). Published by } \\
\text { Cell Physiol Biochem Press GmbH\&Co. KG }\end{array}$ \\
\cline { 2 - 3 }
\end{tabular} \\ Ou et al.: Microvasculature Stabilization through Hypoxic Preconditioning}

positive intercellular signal across the HRMEC monolayer (Fig. 5D-E). Whilst the extent of vascular leak was slightly reduced with conditioned medium from HP-1 hour and 4 hours, this was not statistically significant.

Hypoxic Preconditioning Suppresses Hypoxia-Induced MIO-M1 cells Activation through Regulation of Metabolic Signaling

As a key transcription factor, HIF- $1 \alpha$ also upregulates genes involved in glycolytic energy metabolism and cell survival, including GLUT1 and several glycolytic enzymes [22]. Stabilization of HIF- $1 \alpha$ under normoxia impairs the TCA cycle and mitochondrial activity [23]. We therefore hypothesized that HP could protect mitochondrial function by modulating oxidative metabolism, and thus preventing the HIF-1a mediated glycolysis as a rapid energy source.

To understand how HP may inhibit stabilization of HIF-1 $\alpha$, we examined changes to the mammalian target of rapamycin (mTOR) signaling pathway that is known to regulate HIF [24]. Accordingly, we noted that mTOR and HIF are upregulated in response to hypoxia in MIO-M1 cells, and expression of both is diminished upon pretreatment with rapamycin (mTOR inhibitor) (Fig. 6A). A similar reduction in mTOR and HIF expression is observed in cells subjected to the HP-2 hours protocol (Fig. 6B).

To this end we explored how hypoxia vs HP influenced cellular metabolism. In response to prolonged hypoxia, MIO-M1 cells switch to aerobic glycolysis, shown by the increased expression of GLUT1, and decreased expression of SDHD (Fig. 6C-D). Therefore, switch to aerobic glycolysis under hypoxia is associated with the upregulation of HIF- $1 \alpha$. Interestingly, HP-2 hours prevents this switch to glycolysis as shown by reduced GLUT1 and increased SDHD expression.

Using the mitochondrial stress test confirmed that cells under hypoxia have reduced basal and maximal respiration, as shown with oxygen consumption rate results. This demonstrates that cells have increased aerobic glycolysis compared to mitochondrial activity, which is consistent with the expression profile of metabolic genes GLUT1 and SHDH (Fig. 6E). Interestingly, HP-2 hours partially protected cells' mitochondrial function, as cells after 2 hours HP measured significantly increased basal and maximal respiration, as well as spare respiratory capacity compared to cells not exposed to preconditioning/ under hypoxia (Fig. 6F-H).

Therefore, the mitochondrial stress showed the increased mitochondrial activity after hypoxic preconditioning, which supports the reduced glycolytic function observed by the reduced mRNA expression of key glycolytic enzyme GLUT1.

\section{Discussion}

The protective role that HP offers to tissue, including the retina, has been previously highlighted [25]. Whilst the hypoxic response of MIO-M1 cells promotes vascular permeability through HIF-dependent upregulation of pro-angiogenic factors (e.g ANGPTL4) [16], the potential protective effects of HP have not previously been investigated in those cells. In the current study, we demonstrate that HP has a robust effect decreasing HIF-1 $\alpha$ stabilization in response to hypoxia. In turn, HIF-mediated transcription and secretion of pro-angiogenic factors are reduced. This protective mechanism is linked with a change in the MIO-M1 cells' metabolic status.

Consistent with previous reports [16-21], we have shown that conditioned medium from hypoxic MIO-M1 cells upregulates HIF- $1 \alpha$ and promotes endothelial cell angiogenesis and vascular permeability in HUVEC cultures. Whilst the endothelial cells are from different origins it provides principal of an endothelial non-specificity as well as regulation of tight junctions in common between the two cell types. Furthermore, our data demonstrate a protective effect of HP in hypoxic MIO-M1 cells. The protective effect is consistent with other data showing that hypoxia-preconditioned cells delay apoptosis in response to subsequent 


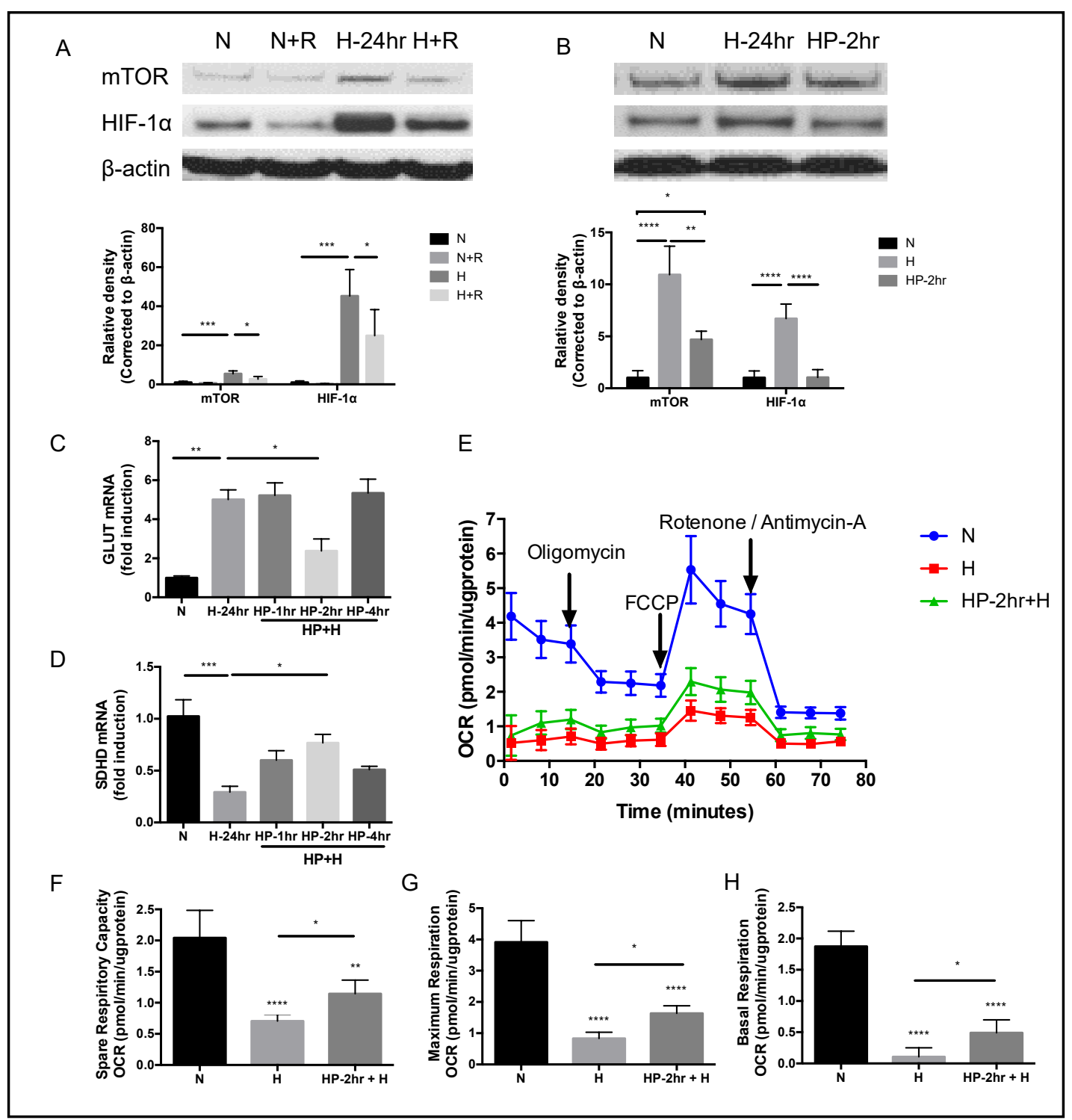

Fig. 6. Hypoxic preconditioning inhibits HIF-1 $\alpha$ activation by modulating mTOR and decreasing glycolysis in MIO-M1 cells. (A) Exposure of MIO-M1 cells to $1 \% \mathrm{O}_{2}$ hypoxia 24 hours ( $\mathrm{H}-24 \mathrm{hr}$ ), the increased expression of mTOR in accordance with HIF- $1 \alpha$ compare to normoxia $(\mathrm{N})$ and both (Normoxia with Rapamycin: N+R; Hypoxia with Rapamycin: $\mathrm{H}+\mathrm{R})$ were inhibited through pretreatment with rapamycin (100 $\mathrm{nM})$ at protein level ( $n=3$ per group). (B) HP-2 hours (HP-2hr) could inhibit the expression of HIF-1 $\alpha$ through suppressing mTOR expression, decreased glycolysis and improves mitochondrial function in comparison with hypoxic treatment ( $n=3$ per group). (C, D) Exposure MIO-M1 cells to hypoxia increased gene expression of GLUT and SDHD compared to normoxic treatment. However, HP-2 hours partially reversed the expression in comparison with hypoxia ( $n=4$ per group). (E) Representative mitochondrial stress test measured with sequential injections of oligomycin, FCCP and rotenone/antimycin A. (F) Spare-respiratory capacity was calculated as the difference between maximal and basal OCR. (G) Maximal respiration was calculated as difference between maximal OCR value post-injection of FCCP and non-mitochondrial OCR. (H) Basal respiration was calculated as the difference between first OCR measurement and non-mitochondrial OCR. Data represents means \pm SD of relative values vs control from 3 independent experiments. $\mathrm{P}<0.05$; ${ }^{* *} \mathrm{P}<0.01$; ${ }^{* * *} \mathrm{P}<0.001 ;{ }^{* * * *} \mathrm{P}<0.0001$, statistical analysis was performed with one-way ANOVA with Dunn's test for multiple comparisons. 


\section{Cellular Physiology Cell Physiol Biochem 2019;52:668-680 \\ \begin{tabular}{ll|l} 
and Biochemistry & $\begin{array}{l}\text { DOl: 10.33594/000000047 } \\
\text { Published online: 29 March 2019 }\end{array}$ & $\begin{array}{l}\text { O } 2019 \text { The Author(s). Published by } \\
\text { Cell Physiol Biochem Press GmbH\&Co. KG }\end{array}$ \\
\cline { 2 - 3 }
\end{tabular} \\ Ou et al.: Microvasculature Stabilization through Hypoxic Preconditioning}

hypoxia [26], hypoxia-preconditioned bone marrow stem cells promote nerve regeneration in cerebral ischemic rats [27], HP treated astrocytes are tolerant to severe hypoxia [19] and HP treated neurons were protected against the effects of subsequent oxygen and glucose deprivation [28]. In our experiments, we show that cells subjected to HP demonstrate improved tolerance to a subsequent severe hypoxic exposure. However, HP as a brief period of hypoxia to induce protection against subsequent severe insult is ill-defined. We assessed the time-course of a cellular response under hypoxia (Fig. 3B-E), and MIO-M1 cells start showing a response at 2 hours which exaggerates at 4 hours. The protective effect of HP on the permeability and angiogenesis assays was optimal for 2 hours, implying the importance of selecting the appropriate duration of HP.

It has been suggested that metabolic products, specifically fumarate and succinate, regulate the expression of hypoxia inducible genes including HIF-1 $\alpha$ in cancer pathology [29]. We therefore assessed metabolic pathways as a potential mechanism through which HP-2 hours exerts the protective anti-angiogenic effect. We found that transcriptional activity of HIF during hypoxia and HIF- $1 \alpha$ stabilization is dependent on the activation of mTOR. Although the mechanism of how mTOR modulates HIF within cells remains elusive, our results confirm previous cancer studies that showed that mTOR activation potently enhances the activity of HIF- $1 \alpha$ and VEGF-A secretion during hypoxia, and this is reversed with rapamycin [24]. In our experiments, HP significantly perturbed mTOR expression suggesting that HP could provide a suitable strategy for the treatment of hypoxic retinopathy by suppressing mTOR-mediated pro-angiogenic factors through HIF- $1 \alpha$.

Furthermore, Lu et al. demonstrated that the upregulation of end products of glycolytic metabolism under hypoxia in cancer - the well-known Warburg effect -promotes HIF-1 $\alpha$ protein stability and activation of HIF-1-inducible genes [22]. In our experiments, we found reduced oxidative metabolism of retinal MIO-M1 cells under hypoxia, which was partially prevented after HP, as shown by oxygen consumption rate results at the mitochondrial stress test (Fig. 6E-H). Furthermore, gene expression of glycolytic proteins was reduced by HP, while SHDH expression was significantly increased, suggesting the usage of the TCA cycle. We observed a correlation of GLUT expression levels with HIF-1 $\alpha$ expression in MIO-M1 cells, with HP preventing the upregulation of GLUT, and subsequently of HIF- $1 \alpha$. Overall, we demonstrate that HP regulates the cellular metabolism of MIO-M1 cells, stabilizing the cells, preventing the switch to aerobic glycolysis, and therefore preventing angiogenesis (Fig. 6CD). Although we propose a mechanism for the protective effect of HP on angiogenesis, future studies are required to investigate further how HP specifically influences all metabolic pathways and the HIF regulation.

The inhibition of HIF- $1 \alpha$ and HIF- $1 \alpha$ - mediated genes expression has emerged as a major target for cancer treatment. Additionally, the beneficial effects of HP in reducing tissue damage from cerebral ischemia are also considered to involve HIF- $1 \alpha$ - mediated genes expression [30]. Our data on retinal MIO-M1 cells and in vitro angiogenesis models further demonstrated that treatments that regulate aerobic glycolysis may also target HIF-1 $\alpha$, with HP representing a potential therapeutic strategy in hypoxic retinopathy. In conclusion, HP induced significant protection against hypoxic insults and suppressed HIF- $1 \alpha$ activation. Pretreatment of MIO-M1 cells with sublethal exposure to hypoxia inhibited the expression of proangiogenic factors reducing the promotion of endothelial cell proliferation and leakage. These results further indicate the importance of HP in preventing hypoxic injury.

\section{Acknowledgements}

This work was supported by The Dunhill Medical Trust [grant number: R474/0216] and China Scholarships Council (CSC). 


\section{Cellular Physiology Cell Physiol Biochem 2019;52:668-680 \begin{tabular}{ll|l} 
and Biochemistry & $\begin{array}{l}\text { DOl: 10.33594/000000047 } \\
\text { Published online: 29 March 2019 }\end{array}$ & $\begin{array}{l}\text { O } 2019 \text { The Author(s). Published by } \\
\text { Cell Physiol Biochem Press GmbH\&Co. KG }\end{array}$ \\
\cline { 2 - 3 }
\end{tabular} \\ Ou et al.: Microvasculature Stabilization through Hypoxic Preconditioning}

\section{Disclosure Statement}

The authors declare that there is no conflict of interest regarding the publication of this paper.

\section{References}

- 1 Kaur C, Foulds WS, Ling EA: Hypoxia-ischemia and retinal ganglion cell damage. Clin Ophthalmol 2008;2:879-89.

2 Nentwich MM, Ulbig MW: Diabetic retinopathy - ocular complications of diabetes mellitus. World J Diabetes 2015;6:489-99.

3 Bek T: Inner retinal ischaemia: current understanding and needs for further investigations. Acta Ophthalmol 2009;87:362-367.

4 Bernaudin M, Nedelec AS, Divoux D, MacKenzie ET, Petit E, Schumann-Bard P: Normobaric Hypoxia Induces Tolerance to Focal Permanent Cerebral Ischemia in Association with an Increased Expression of HypoxiaInducible Factor- 1 and its Target Genes, Erythropoietin and VEGF, in the Adult Mouse Brain. J Cereb Blood Flow Metab 2002;22:393-403.

- 5 Maxwell PH: Hypoxia-inducible factor as a physiological regulator. Exp Physiol 2005;90:791-797.

- 6 Schofield CJ, Ratcliffe PJ: Oxygen sensing by HIF hydroxylases. Nat Rev Mol Cell Biol 2004;5:343-354.

7 Semenza GL: Hydroxylation of HIF-1: Oxygen Sensing at the Molecular Level. Physiology 2004;19:176-182.

- 8 Semenza GL: Hypoxia-inducible factors in physiology and medicine. Cell 2012;148:399-408.

- 9 Bruick RK, McKnight SL: TRANSCRIPTION: Enhanced: Oxygen Sensing Gets a Second Wind. Science 2002;295:807-808.

- 10 Cheng L, Yu H, Yan N, Lai K, Xiang M: Hypoxia-Inducible Factor-1 $\alpha$ Target Genes Contribute to Retinal Neuroprotection. Front Cell Neurosci 2017;11:20.

- 11 Grimm C, Willmann G: Hypoxia in the Eye: A Two-Sided Coin. High Alt Med Biol 2012;13:169-175.

- 12 Willmann G, Fischer MD, Schatz A, Schommer K, Gekeler F: Retinal Vessel Leakage at High Altitude. JAMA 2013;309:2210-2212.

13 Vecino E, Rodriguez FD, Ruzafa N, Pereiro X, Sharma SC: Glia-neuron interactions in the mammalian retina. Prog Retin Eye Res 2016;51:1-40.

14 Newman EA: Acid efflux from retinal glial cells generated by sodium bicarbonate cotransport. J Neurosci 1996;16:159-168.

- 15 Bringmann A, Pannicke T, Grosche J, Francke M, Wiedemann P, Skatchkov SN, Osborne NN, Reichenbach A: Müller cells in the healthy and diseased retina. Prog Retin Eye Res 2006;25:397-424.

- 16 Xin X, Rodrigues M, Umapathi M, Kashiwabuchi F, Ma T, Babapoor-Farrokhran S, Wang S, Hu J, Bhutto I, Welsbie DS, Duh EJ, Handa JT, Eberhart CG, Lutty G, Semenza GL, Montaner S, Sodhi A: Hypoxic retinal Muller cells promote vascular permeability by HIF-1-dependent up-regulation of angiopoietin-like 4. Proc Natl Acad Sci U S A 2013;110:E3425-3434.

- 17 Babapoor-Farrokhran S, Jee K, Puchner B, Hassan SJ, Xin X, Rodrigues M, Kashiwabuchi F, Ma T, Hu K, Deshpande M, Daoud Y, Solomon S, Wenick A, Lutty GA, Semenza GL, Montaner S, Sodhi A: Angiopoietinlike 4 is a potent angiogenic factor and a novel therapeutic target for patients with proliferative diabetic retinopathy. Proc Natl Acad Sci U S A 2015;112:E3030-3039.

- 18 Stetler RA, Leak RK, Gan Y, Li P, Zhang F, Hu X, Jing Z, Chen J, Zigmond MJ, Gao Y: Preconditioning provides neuroprotection in models of CNS disease: Paradigms and clinical significance. Prog Neurobiol 2014;114:58-83.

19 Liu M, Alkayed NJ: Hypoxic Preconditioning and Tolerance via Hypoxia Inducible Factor (HIF) $1 \alpha$-linked Induction of $P 450$ 2C11 Epoxygenase in Astrocytes. J Cereb Blood Flow Metab 2005;25:939-948.

20 Kast B, Schori C, Grimm C: Hypoxic preconditioning protects photoreceptors against light damage independently of hypoxia inducible transcription factors in rods. Exp Eye Res 2016;146:60-71.

- 21 Rodrigues M, Xin X, Jee K, Babapoor-Farrokhran S, Kashiwabuchi F, Ma T, Bhutto I, Hassan SJ, Daoud Y, Baranano D, Solomon S, Lutty G, Semenza GL, Montaner S, Sodhi A: VEGF secreted by hypoxic Müller cells induces MMP-2 expression and activity in endothelial cells to promote retinal neovascularization in proliferative diabetic retinopathy. Diabetes 2013;62:3863-3873. 


\section{Cellular Physiology and Biochemistry}

Cell Physiol Biochem 2019;52:668-680

22 Lu H, Forbes RA, Verma A: Hypoxia-inducible factor 1 activation by aerobic glycolysis implicates the Warburg effect in carcinogenesis. J Biol Chem 2002;277:23111-23115.

- 23 Selak MA, Armour SM, MacKenzie ED, Boulahbel H, Watson DG, Mansfield KD, Pan Y, Simon MC, Zhompson CB, Gottlieb E: Succinate links TCA cycle dysfunction to oncogenesis by inhibiting HIF- $\alpha$ prolyl hydroxylase. Cancer Cell 2005;7:77-85.

- 24 Land SC, Tee AR: Hypoxia-inducible factor 1alpha is regulated by the mammalian target of rapamycin (mTOR) via an mTOR signaling motif. J Biol Chem 2007;282:20534-20543.

- 25 Grimm C, Hermann DM, Bogdanova A, Hotop S, Kilic Ü, Wenzel A, Kilic E, Gassmann M: Neuroprotection by hypoxic preconditioning: HIF-1 and erythropoietin protect from retinal degeneration. Semin Cell Dev Biol 2005;16:531-538.

26 Chen O, Wu M, Jiang L: The Effect of Hypoxic Preconditioning on Induced Schwann Cells under Hypoxic Conditions. PLoS One 2015;10:e0141201.

27 Roth S, Dreixler JC, Mathew B, Balyasnikova I, Mann JR, Boddapati V, Xue L, Lesniak MS: HypoxicPreconditioned Bone Marrow Stem Cell Medium Significantly Improves Outcome After Retinal Ischemia in Rats. Invest Ophthalmol Vis Sci 2016;57:3522-3532.

28 Pacary E, Legros H, Valable S, Duchatelle P, Lecocq M, Petit E, Nicole O, Bernaudin M: Synergistic effects of $\mathrm{CoCl} 2$ and ROCK inhibition on mesenchymal stem cell differentiation into neuron-like cells. J Cell Sci 2006;119:2667-2678.

- 29 Laukka T, Mariani CJ, Ihantola T, Cao JZ, Hokkanen J, Kaelin WG Jr, Godley LA, Koivunen P: Fumarate and Succinate Regulate Expression of Hypoxia-inducible Genes via TET Enzymes. J Biol Chem 2016;291:42564265 .

- 30 Sharp FR, Ran R, Lu A, Tang Y, Strauss KI, Glass T, Ardizzone T, Bernaudin M: Hypoxic preconditioning protects against ischemic brain injury. NeuroRx 2004;1:26-35. 